\section{RMD Open}

Rheumatic \&

Musculoskeletal Diseases

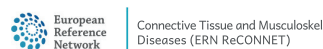

\title{
Undifferentiated connective tissue disease: state of the art on clinical practice guidelines
}

Margarida Antunes, ${ }^{1}$ Carlo Alberto Scirè, ${ }^{\oplus 2}$ Rosaria Talarico, ${ }^{3}$ Tobias Alexander, ${ }^{\odot 4}$ Tadej Avcin, ${ }^{5}$ Chiara Belocchi, ${ }^{6}$ Andrea Doria, ${ }^{7}$ Franco Franceschini, ${ }^{8}$ Ilaria Galetti, ${ }^{9}$ Marcello Govoni, ${ }^{10}$ Eric Hachulla, ${ }^{11}$ David Launay, ${ }^{11}$ Gemma Lepri, ${ }^{12}$ Carla Macieira, ${ }^{13}$ Marco Matucci-Cerinic, ${ }^{12}$ Carlo Maurizio Montecucco, ${ }^{14}$ Maria Francisca Moraes-Fontes, ${ }^{\circ}$ Luc Mouthon, ${ }^{15}$ Sabrina Paolino, ${ }^{16}$ Veronique Ramoni, ${ }^{14}$ Chiara Tani, ${ }^{3}$ Sander W Tas, ${ }^{17}$ Angela Tincani, ${ }^{8}$ Ronald Van Vollenhoven, ${ }^{18}$ Margherita Zen, ${ }^{7}$ Joao Fonseca, ${ }^{19}$ Stefano Bombardieri, ${ }^{19}$ João Eurico Fonseca, ${ }^{13}$ Matthias Schneider, ${ }^{20}$ Vanessa Smith, ${ }^{21,22}$ Maurizio Cutolo, ${ }^{16}$ Marta Mosca, ${ }^{3,23}$ Lorenzo Beretta $^{\bullet}$

To cite: Antunes M, Scirè CA, Talarico R, et al. Undifferentiated connective tissue disease: state of the art on clinical practice guidelines. RMD Open 2019;4:e000786. doi:10.1136/ rmdopen-2018-000786

Received 31 July 2018 Revised 27 December 2018 Accepted 2 January 2019
Check for updates

(C) Author(s) (or their employer(s)) 2019. Re-use permitted under CC BY-NC. No commercial re-use. See rights and permissions. Published by BMJ.

For numbered affiliations see end of article.

Correspondence to Dr Lorenzo Beretta; lorberimm@hotmail.com

\section{ABSTRACT}

The term 'undifferentiated connective tissue disease' (UCTD) is generally used to describe clinical entities characterised by clinical and serological manifestations of systemic autoimmune diseases but not fulfilling the criteria for defined connective tissue diseases (CTDs). In this narrative review, we summarise the results of a systematic literature research, which was performed as part of the ERN ReCONNET project, aimed at evaluating existing clinical practice guidelines (CPGs) or recommendations. No specific CPG on UCTD were found, potential areas of intervention are absence of a consensus definition of UCTD, need for specific monitoring and therapeutic protocols, stratification of UCTD based on the risk of developing a defined CTD and preventive measure for the future development of a more severe condition.

Patients feel uncertainty regarding the name of the disease and feel the need of a better education and understanding of these conditions and its possible changes over time.

\section{INTRODUCTION}

The term 'undifferentiated connective tissue disease' (UCTD) is generally used to describe clinical entities characterised by serological evidence of autoimmunity and by the occurrence of clinical symptoms often seen in association with systemic autoimmune diseases (SADs), yet not fulfilling criteria for a specific SAD. Compared with other SADs, whose classifications are formally issued by rheumatological societies and periodically subject to discussion and revisions, the overall concept of UCTD may seem quite vague and prone to (mis) interpretation. As such, clinicians may be tempted to classify early phases of other

\section{Key messages}

What is already known about this subject?

- Undifferentiated Connective Tissue Diseases (UCTD) is an orphan rheumatological condition often observed in clinical practice.

- No guideline on UCTD diagnosis and management is widely accepted or routinely used.

What does this study add?

- An extensive literature search by the ERN ReCONNECT team could not identify any guideline or recommendation on UCTD.

How might this impact on clinical practice?

- Clinicians are concerned about the lack of a proper definition of UCTD, risk assessment as well as monitoring and therapeutic strategies for patients with UCTD.

- Patients with UCTD may feel uncertainty regarding UCTD diagnosis, therapeutic approach and prognosis.

major SADs as UCTD. Not surprisingly, literature data show that up to $30 \%$ of UCTD may evolve into a definite 'major' SAD within few years from the diagnosis (all reviewed in ${ }^{1}$ ). The use of the term UCTD to describe an early phase of a major rheumatic disease and patient who temporarily lack sufficient features to meet other classification criteria was first proposed by LeRoy et al in $1980 .^{2}$ To best distinguish between UCTD at risk of evolution and stable UCTD, preliminary, yet often adopted, criteria for UCTD were proposed in $1999 .{ }^{3}$ Patients can be classified as UCTD if they fulfil the criteria as follows: 
(1) signs and symptoms suggestive of a connective tissue disease (CTD), but not fulfilling criteria for a defined CTD, (2) positive antinuclear antibodies on two separate measurements controls and (3) a disease duration of at least 3 years. These criteria strongly rely on the absence of other SADs, that is the inability to fulfil other classification criteria for major CTDs. During the last decade, classification criteria for other major SADs have been subject of extensive review in the attempt to make them more sensitive and capable of capturing early phases of CTDs. ${ }^{4-8}$ This process is actively ongoing and is likely to produce new sets of criteria in the very near future. ${ }^{9}$ It is clear that any new or revised criteria for SADs may affect our view of UCTD and change the 'label' clinicians tag to patients with early autoimmune diseases. Still, it is unclear to what extent this improved knowledge will ultimately affect the clinical practice and the clinicians' attitude at bedside. The current knowledge on disease evolution in patients with UCTD and the factors that may influence the progression towards a definite SAD may be affected as well. ${ }^{10}$ These challenges are inflated considering the limited performance classification criteria may have when used as diagnostic tools in absence of proper diagnostic criteria. ${ }^{11}$ On the other hand, diagnostic criteria for UCTD may be difficult to develop in absence of clear-cut definitions and if too strict they may limit the ability to make a clinical diagnosis and preclude the patients a proper treatment and follow-up.

The present work tries to identify the current clinical practice guidelines (CPGs) in UCTD and the clinicians' and patients' unmet needs that come along with the diagnosis and changing definition of UCTD.

\section{METHODS}

\section{Search for existing guidelines}

We carried out a systematic search in PUBMED and EMBASE based on controlled terms (MeSH and Emtree, see below) and keywords of the disease and publication type (CPGs). Two independent reviewers (RT, CAS) screened all the retrieved articles applying the following inclusion criteria: UCTD (population) and CPGs on the area of diagnosis, monitoring and treatment (study design). CPG were defined according to the Institute of Medicine 2011 definition (Clinical practice guidelines are statements that include recommendations intended to optimise patient care that are informed by a systematic review of evidence and an assessment of the benefits and harms of alternative care options). No language or year of publication restriction was applied.

The Disease Coordinators (DC) of the ERNReCONNET for UCTD (LB, MA) first performed a screening among papers included in the final list (systematic) based on Title and Abstract selected evidence-based medicine guidelines; second, they performed a hand search looking for existing guidelines in selected sites from national clinical societies and through multiple search engines (Google and Bing). Prespecified data extraction included author, year, area, clinical question coverage and AGREE II domains (Scope and Purpose; Stakeholder Involvement; Rigour of Development; Clarity of Presentation; Applicability; Editorial Independence. Data were planned to be reported descriptively by area (diagnosis, monitoring and management), to describe coverage and to inform the unmet need phase.

\section{Medline (Pubmed) terms}

(undifferentiated(All Fields) AND ('connective tissue diseases'(MeSH Terms) OR ('connective'(All Fields) AND 'tissue'(All Fields) AND 'diseases'(All Fields) ) OR 'connective tissue diseases' (All Fields) OR ('connective' (All Fields) AND 'tissue' (All Fields) AND 'disease'(All Fields) ) OR 'connective tissue disease'(All Fields))) AND ('Practice Guideline'(Publication Type) OR 'Practice Guidelines As Topic' (MeSH Terms) OR Practice Guideline(Publication Type) OR 'Practice Guideline'(Text Word) OR 'Practice Guidelines' (Text Word) OR 'Guideline'(Publication Type) OR 'Guidelines As Topic'(MeSH Terms) OR Guideline(Publication Type) OR 'Guideline'(Text Word) OR 'Guidelines' (Text Word) OR 'Consensus Development Conference'(Publication Type) OR 'Consensus Development Conferences As Topic'(MeSH Terms) OR 'Consensus'(MeSH Terms) OR 'Consensus'(Text Word) OR 'Recommendation'(Text Word) OR 'Recommendations'(Text Word) OR 'Best Practice' (Text Word) OR 'Best Practices' (Text Word)).

\section{Embase}

('undifferentiated connective tissue disease'/exp OR ('connective tissue disease'/ $\exp$ OR 'connective tissue disease' OR 'connective tissue diseases' OR 'connective tissue disorder' AND undifferentiated)) AND ('practice guideline'/exp OR 'practice guideline' OR 'practice guidelines'/exp OR 'practice guidelines' OR 'clinical practice guideline'/exp OR 'clinical practice guideline' OR 'clinical practice guidelines' / exp OR ‘clinical practice guidelines' OR 'clinical practice guidelines as topic'/exp OR 'clinical practice guidelines as topic' OR 'guideline' / exp OR 'guideline' OR 'guidelines' / exp OR 'guidelines' OR 'guidelines as topic'/exp OR 'guidelines as topic' OR 'consensus development' / exp OR 'consensus development' OR 'consensus development conference'/exp OR 'consensus development conference' OR 'consensus development conferences' / exp OR 'consensus development conferences' OR 'consensus development conferences as topic'/ exp OR 'consensus development conferences as topic' OR 'consensus'/exp OR 'consensus' OR 'recommendation' OR 'recommendations') AND (embase)/lim NOT (medline) /lim

\section{Identification of patient's unmet needs}

Patients and families referring to the ERN ReCONNET European Patients Advocacy Group (ePAG) as well as 
caregivers have been deeply involved in the ERN activities and their personal views on UCTD unmet needs have been expressed during web conferences and meetings. The collected needs have been reviewed and rephrased by the patients' representative and herein presented in a simplified and narrative form.

\section{RESULTS}

\section{Identification of existing CPGs}

Overall 108 articles were considered eligible for review after search in PUBMED and EMBASE. All of them were independently reviewed by LB and MA; both the reviewers agreed that none of the selected article contained suitable CPGs for further assessment. Similarly, the hand search via search engines and in selected websites did not yield any publication matching with inclusion criteria The overall result is that no CPG guideline on UCTD is currently publicly available.

\section{Clinicians' unmet needs}

From literature and hand search, it is clear that several aspects of UCTD are still vague and as such we can identify several potential area of intervention or interest for the clinical practitioners.

1. The lack of CPGs on UCTD may reflect the lack of a consensus definition on UCTD. To date UCTD classification criteria are only provisional and may be considered outdated in light of the new SADs criteria that capture with increased sensitivity early or even preclinical CTD in patients. We can thus identify the necessity to develop, refine and validate the definition of UCTD as a strong need.

2. In the absence of proper guidelines, it is unclear whether patients with UCTD with features resembling major SADs should be followed according to existing protocols and guidelines (ie, those reviewed in the other articles of the Supplement that come along with this work) or if specific guidelines should be developed.

3. It would be of interest to perform a proper stratification of patients with UCTD in relation to the risk of developing specific SADs. These patients could be generally labelled as 'UCTD at risk for [...]' in relation to clinical, serological or other available data. Similarly to what has beforehand been discussed (see point 2), ad hoc guidelines could be developed for such patients or, alternatively, existing ones should be adapted and applied to them.

4. In the absence of rigorous prospective epidemiological data, it is unclear to what extent the early introduction or the use of drugs developed for other indications or commonly prescribed in rheumatology clinical practice may be useful for patients with UCTD. The impact of such therapeutic approach on the natural history of UCTD is unclear. Specific (inter) national registries for UCTD may facilitate collection of these data. ${ }^{12}$

\section{Patients' unmet needs}

This paragraph intends to highlight the unmet needs of the Undifferentiated Connective Tissue Disease European community. The content of this paragraph has been realised by the ERN ReCONNET ePAG that carefully but relentlessly collected the voices and the points of view of the whole European community of the disease they represent. The rarity of the disease and the scarcity of patient organisations made this work harder than expected.

Patients feel uncertainty regarding the name of the disease; the word 'undifferentiated' makes them feel as if they do not have a proper disease, but rather one that only in the future will become a recognised disease. This feeling has a high impact on their health related quality of life. Patients may feel neglected as the mere presence of antinuclear antibody titres do not seem to justify the necessity of consistent diagnostic and follow-ups, which should be better explained. The (low) risk percentage of developing another $\mathrm{SAD}$ and its consequences should be better described, as well as the bigger chance of not developing another SAD. Patients also refer to the lack of information on the disease and report the lack of involvement by healthcare providers in producing the existing documents, maybe due to the scarcity of patients' groups. The lack of CPGs for UCTD seems to substantiate this complaint.

\section{CONCLUSION}

The current lack of available CPGs clearly shows that UCTD is somehow neglected as compared with other major SADs. Despite the possibility that some patients with UCTD may indeed harbour the possibility to progress towards early and definite forms of other CTDs, there is no consensus on the optimal management of these patients. In the absence of clear-cut epidemiological studies and of a thorough risk assessment, individual experience still guides the clinician's choice. As such, patients with UCTD are left within an area of uncertainty despite the generic reassurance that their disease is somehow 'milder' compared with defined SADs. This attitude is clearly a source of discomfort as patients and relatives feel disturbing an uncertain situation that may leave more questions than answers (What do I exactly have? What will happen to me? How will my disease develop?). The uncertainty regarding UCTD diagnosis, therapeutic approach and prognosis may influence patients' coping strategies, discourage a thorough and systematic follow-up and assessment which may have potential drawbacks on the early identification of cases progressing towards definite SADs or development of severe complications. Patients may feel a reduced trust on healthcare providers, which has a direct effect on outcomes of care. ${ }^{13}$ Consequently, focused efforts are clearly needed to integrate the specialist's experience with patients' needs and expectations to foster the development of formal UCTD CPGs.

\section{Author affiliations}

${ }^{1}$ Autoimmune Disease Unit (Unidade de Doenças Auto-imunes/Medicina 7.2), Hospital de Curry Cabral, Centro Hospitalar de Lisboa Central, Lisboa, Portugal 
${ }^{2}$ Section of Rheumatology, Department of Medical Sciences, University of Ferrara, Ferrara, Italy

${ }^{3}$ Rheumatology Unit, AOU Pisana, Pisa, Italy

${ }^{4}$ Department of Rheumatology and Clinical Immunology, Charité University Hospital Berlin, Berlin, Germany

${ }^{5}$ Department of Allergology, Rheumatology and Clinical Immunology, University Children's Hospital, University Medical Centre Ljubljana, Ljubljana, Slovenia ${ }^{6}$ Referral Center for Systemic Autoimmue Diseases, Fondazione IRCCS Ca' Granda Ospedale Maggiore Policlinico di Milano, Milan, Italy

${ }^{7}$ Rheumatology Unit, Department of Medicine, A0 Padova and University of Padua, Padua, Italy

${ }^{8}$ Rheumatology and Clinical Immunology Unit, Civil Hospital, Brescia, Italy

${ }^{9} \mathrm{FESCA}$, Federation of European Scleroderma Associations, Milan, Italy

${ }^{10}$ Infectious Diseases Unit, Department of Medical Sciences, University of Ferrara and S Anna University Hospital, Ferrara, Italy

${ }^{11}$ Département de Médecine Interne et Immunologie Clinique, Centre de Référence des Maladies Systémiques et Auto-Immunes Rares du Nord-Ouest (CERAINO), LIRIC, INSERM, Univ Lille, CHU Lille, Lille, France

${ }^{12}$ Department of Clinical and Experimental Medicine, Division of Rheumatology and Scleroderma Unit, AOU Careggi, University of Florence, Florence, Italy

${ }^{13}$ Rheumatology Department, Hospital de Santa Maria, Centro Hospitalar Lisboa Norte, Lisbon Academic Medical Centre, Libson, Portugal

${ }^{14}$ Division of Rheumatology, University and IRCCS Policlinico S. Matteo Foundation, Pavia, Italy

${ }^{15}$ Service de Médecine Interne, Hôpital Cochin, Centre de Référence Maladies systémiques Autoimmunes Rares d'lle de France, Assistance Publique-Hôpitaux de Paris (AP-HP), Université Paris Descartes, Paris, France

${ }^{16}$ Research Laboratory and Academic Division of Clinical Rheumatology, Department of Internal Medicine, IRCCS Polyclinic Hospital San Martino, University of Genoa, Genoa, Italy

${ }^{17}$ Amsterdam Rheumatology \& Immunology Center (ARC), Academic Medical Center, Amsterdam, The Netherlands

${ }^{18}$ Clinical Immunology \& Rheumatology, Amsterdam Rheumatology \& Immunology Center, Academic Medical Center/University of Amsterdam, Amsterdam, The Netherlands

${ }^{19}$ University of Pisa, Pisa, Italy

${ }^{20}$ Department of Rheumatology, Universitätsklinikum Düsseldorf, Düsseldorf, Germany

${ }^{21}$ Department of Rheumatology, Ghent University Hospital, Ghent, Belgium

${ }^{22}$ Department of Internal Medicine, Ghent University, Ghent, Belgium

${ }^{23}$ Rheumatology Unit, University of Pisa, Pisa, Italy

Acknowledgements Thanks to all the members of the Steering Committee of the ERN ReCONNET for the huge commitment during this work. A special thanks to all the members of the ERN ReCONNET team for providing support during all the phases of the Work Package 3.

Contributors All the authors critically reviewed and approved the manuscript and gave a substantial contribution to its drafting.

Funding This publication was funded by the European Union's Health Programme (2014-2020)

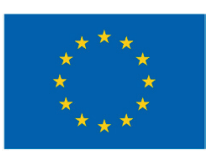

Disclaimer ERN ReCONNET is one of the 24 European Reference Networks (ERNs) approved by the ERN Board of Member States. The ERNs are co-funded by the European Commission. The content of this publication represents the views of the authors only and it is their sole responsibility; it cannot be considered to reflect the views of the European Commission and/or the Consumers, Health, Agriculture and Food Executive Agency (CHAFEA) or any other body of the European Union. The European Commission and the Agency do not accept any responsibility for use that may be made of the information it contains.
Competing interests None declared.

Patient consent Not required.

Provenance and peer review Commissioned; externally peer reviewed.

Data sharing statement № additional data are available.

Open access This is an open access article distributed in accordance with the Creative Commons Attribution Non Commercial (CC BY-NC 4.0) license, which permits others to distribute, remix, adapt, build upon this work non-commercially, and license their derivative works on different terms, provided the original work is properly cited, appropriate credit is given, any changes made indicated, and the use is non-commercial. See: http://creativecommons.org/licenses/by-nc/4.0/

\section{REFERENCES}

1. Mosca M, Tani C, Talarico R, et al. Undifferentiated connective tissue diseases (UCTD): simplified systemic autoimmune diseases. Autoimmun Rev 2011;10:256-8.

2. LeRoy EC, Maricq HR, Kahaleh MB, Bashar Kahaleh M. Undifferentiated connective tissue syndromes. Arthritis Rheum 1980;23:341-3

3. Mosca M, Neri R, Bombardieri S. Undifferentiated connective tissue diseases (UCTD): a review of the literature and a proposal for preliminary classification criteria. Clin Exp Rheumatol 1999;17:615-20.

4. Aletaha D, Neogi T, Silman AJ, et al. 2010 rheumatoid arthritis classification criteria: an American college of rheumatology/ european league against rheumatism collaborative initiative. Arthritis \& Rheumatism 2010;62:2569-81.

5. van den Hoogen F, Khanna D, Fransen J, et al. 2013 classification criteria for systemic sclerosis: an American college of rheumatology/ european league against rheumatism collaborative initiative. Arthritis \& Rheumatism 2013;65:2737-47.

6. Shiboski CH, Shiboski SC, Seror R, et al. 2016 American College of Rheumatology/European League against rheumatism classification criteria for primary Sjögren's syndrome: a consensus and datadriven methodology involving three international patient cohorts. Arthritis Rheumatol 2017;69:35-45.

7. Lundberg IE, Tjärnlund A, Bottai M. International myositis classification criteria project consortium, the euromyositis register, and the juvenile dermatomyositis cohort biomarker study and repository (UK and ireland). European league against rheumatism/ american college of rheumatology classification criteria for adult and juvenile idiopathic inflammatory myopathies and their major subgroups. Ann Rheum Dis 2017;76:1955-64.

8. Alarcón-Segovia D, Cardiel MH. Comparison between 3 diagnostic criteria for mixed connective tissue disease. study of 593 patients. $J$ Rheumatol 1989;16:328-34.

9. Johnson S. Presentation at: ACR/ARHP 2017 annual meeting;. European league against rheumatism and American college of rheumatology present new SLE classification criteria at the 2017 ACR/ARHP annual meeting, San Diego, CA, November 3-8, 2017.

10. García-González M, Rodríguez-Lozano B, Bustabad S, et al. Undifferentiated connective tissue disease: predictors of evolution into definite disease. in addition, the high prevalence of anti-DFS70 antibodies in the UCTD patients suggested the potential role of these autoantibodies as a marker in the evolution of UCTD to CTD. Clin Exp Rheumatol 2017;35:739-45

11. Aggarwal R, Ringold S, Khanna D, et al. Distinctions between diagnostic and classification criteria? Arthritis Care Res 2015;67:891-7.

12. Musters A, Tas SW. How to monitor safety and efficacy of biologic treatment in rare, therapy-refractory immune-mediated inflammatory diseases? Rheumatology 2018;57:591-3.

13. Robinson CA. Trust, health care relationships, and chronic illness: a theoretical coalescence. Glob Qual Nurs Res 2016;3:2333393616664823. 